\title{
Foreword to the special issue of CAD/Graphics 2011
}

\author{
Ralph Martin · Hiromasa Suzuki • Changhe Tu
}

Published online: 16 March 2013

(C) Springer-Verlag Berlin Heidelberg 2013

The 12th International Conference on Computer-Aided Design and Computer Graphics (CAD/Graphics 2011) was held in Shandong University, Jinan, China, on September 15-17, 2011, and was sponsored by the China Computer Federation. The conference provided an international forum for researchers and developers from various countries to exchange new ideas on computer aided design and computer graphics.

Initially eight papers were selected from the best papers accepted for the conference and their authors submitted an extended version to the special issue. Each submission was assigned to at least three reviewers and underwent at least two review cycles, in addition to the conference reviewing. In the end five papers were accepted for the special issue.

In paper [1], the authors present a skeleton-driven deformation method; they propose constructing a lattice of cubic cells embracing the input surface mesh to drive the deformation. A novel method is presented in paper [2] for creating a panoramic image of a borehole from a video sequence, without the need for camera calibration and tracking. The authors of paper [3] show how to use movable cellular automata (MCA) to simulate fracture phenomena in hetero-

\section{R. Martin $(\varangle)$}

Computer Science \& Informatics, Cardiff University,

5 The Parade, Roath, Cardiff, CF24 3AA, UK

e-mail: ralph@cs.cf.ac.uk

\section{H. Suzuki}

RCAST, The University of Tokyo, 4-6-1, Komaba, Meguro,

Tokyo 153-8904, Japan

e-mail: suzuki@den.rcast.u-tokyo.ac.jp

\section{Tu}

School of Computer Science and Technology, Shandong University, 1500, The Shunhua Road, Jinan 250101, China e-mail: chtu@sdu.edu.cn geneous objects. Paper [4] presents an expressive rendering approach for visualizing large-scale 3D city scenes, combining photorealistic and nonphotorealistic rendering styles to highlight the information of interest to users while deemphasizing other less important content. A simple and effective method is presented in paper [5] to create volumetric halos to enhance depth perception and display spatial relationships in illustrative visualization.

We would like to thank all the external reviewers, the authors of the selected papers, and others who have helped to ensure the high quality of the papers. We wish to acknowledge Prof. Dr. Nadia Magnenat-Thalmann, Editor-in-Chief of The Visual Computer, for allowing us to produce this special issue and helping with the process, as well as all staff at Springer for their help during the production process.

\section{References}

1. Chen, C.-H., Tsai, M.-H., Lin, I.-C., Lu, P.-H.: Skeleton-driven surface deformation through lattices for real-time character animation. Vis. Comput. 29(4)

2. Niu, C., Zhong, F., Xu, S., Yang, C., Qin, X.: Cylindrical panoramic mosaicing from a pipeline video through MRF based optimization. Vis. Comput. 29(4)

3. Ning, J., Xu, H., Wu, B., Zeng, L., Li, S., Xiong, Y.: Modeling and animation of fracture of heterogeneous materials based on CUDA. Vis. Comput. 29(4)

4. Pan Bin, Zhao, Y., Guo, X., Chen, X., Chen, W., Peng, Q.: Perception-motivated visualization for 3D city scenes. Vis. Comput. 29(4)

5. Tao, Y., Wang, C., Lin, H., Dong, F., Clapworthy, G.: Opacity volume based halo generation and depth-dependent halos. Vis. Comput. 29(4) 


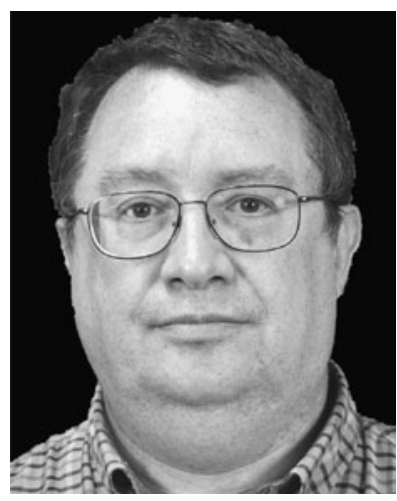

Ralph Martin obtained his $\mathrm{PhD}$ in 1983 from Cambridge University. Since then he has been at Cardiff University, where he now holds a Chair and leads the Visual Computing research group. $\mathrm{He}$ is also a Guest Professor at Tsinghua and two other Universities in China, and Director of Scientific Programmes of the One Wales Research Institute of Visual Computing. His publications include over 200 papers and 12 books covering such topics as solid modelling, surface modelling, reverse engineering, intelligent sketch input, mesh processing, video processing, computer graphics, vision based geometric inspection, and geometric reasoning. He is a Fellow of the Learned Society of Wales, the Institute of Mathematics and its Applications, and the British Computer Society. He is on the editorial boards of "Computer Aided Design", "Computer Aided Geometric Design", "Geometric Models", and several other journals.

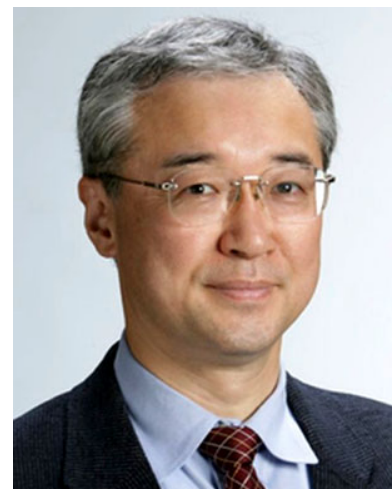

Hiromasa Suzuki is a professor of RCAST (Research Center for Advanced Science and Technologies) at The University of Tokyo. He received his doctor degree in precision machinery engineering from the University of Tokyo in 1986. His research interests include geometric modeling and its applications to digital engineering. Professor Suzuki is a member of the Editorial Board of the Journal of Computer Aided Design and International Journal of $C A D / C A M$. He is a Fellow of JSME (Japan Society for Mechanical Engineers) and a member of JSPE (Japan Society for Precision Engineers), IEEE and ACM.

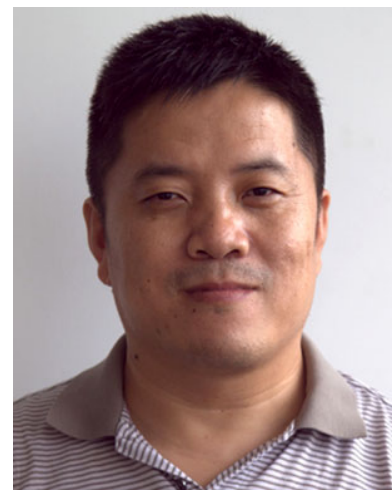

Changhe Tu is a professor of School of Computer Science and Technology at Shandong University. He obtained his $\mathrm{PhD}$ in 2003 from Shandong University. His research interests include geometric modeling and processing, computer aided geometric design, computer graphics and computer animation. His publications cover such topics as analysis and computation of intersections between two quadric surfaces, distance computation of curved surfaces, mesh processing, and facial expression tracking. He is a member of CCF (China Computer Federation) and ACM. 